\title{
Téoros
}

Revue de recherche en tourisme

\section{Quand la concurrence des espaces exige des choix}

\section{En Suède}

\section{Jacques Luneau}

Volume 2, numéro 3, octobre 1983

Les multiples facettes de l'aménagement touristique

URI : https://id.erudit.org/iderudit/1080826ar

DOI : https://doi.org/10.7202/1080826ar

Aller au sommaire du numéro

Éditeur(s)

Université du Québec à Montréal

ISSN

0712-8657 (imprimé)

1923-2705 (numérique)

Découvrir la revue

Citer cet article

Luneau, J. (1983). Quand la concurrence des espaces exige des choix : en Suède.

Téoros, 2(3), 17-19. https://doi.org/10.7202/1080826ar d'utilisation que vous pouvez consulter en ligne.

https://apropos.erudit.org/fr/usagers/politique-dutilisation/ 


\title{
Quand la concurrence des espaces exige des choix
}

\author{
Par Jacques Luneau
}

Si pour des raisons culturelles évidentes, nous entretenons d'étroites relations avec la France et les pays de la communauté francophone, il y a, en matière de tourisme, un pays qui nous ressemble beaucoup: la Suède. La végétation, les conditons clima tiques, les grands espaces, les nombreux lacs sont autant de points qui nous sont similaires.

Une population de 8 millions d'habitants, concentree à $70 \%$ dans les villes dont trois zones métropolitaines importantes: Stockholm, Goteborg, Malmo. Le moyennord et la Laponie à peu près inahabités. Parler d'aménagement touristique en Suède nous oblige à parler de ses rapports étroits avec l'environnement. Les Suédois sont particulièrement sensibles à tout ce qui concerne la nature, l'écologie. Des groupes de pression formés depuis le début du siècle veillent à sa défense. Qui n'a pas entendu parler du "droit d'accès commun" quil qui existe depuis des temps immémoriaux?

Notre but n'est pas tellement de comparer de façon systématique le Québec et la Suède. Si l'amenagement touristique se poursuit de façon quotidienne par les communes locales, avec la création de pistes de ski, de sites pour le camping, de villages de vacances, il n'en demeure pas moins que le rapport du comité d'enquête ${ }^{[2]}$ chargé de la question des résidences secondaires constitue un tournant dans la manière d'aborder le problème. On considérait auparavant, de façon presque idyllique, le fait de pouvoir se rendre dans la nature et de posséder une résidence secondaire. C'est en quelque sorte un trait culturel des Suedois.

Or, la concentration progressive des maisons secondaires dans les espaces les plus propices à la récréation de plein-air et la hausse des coûts posèrent de front la ques: tion de la rentabilité sociale d'un tel développement. Un changement d'orientation était necessaire. Avant de passer aux actions qui ont été entreprises, ces dernières années, voyons un peu les facteurs lourds qui influencent le phénomène du tourisme et du loisir en Suède.

\section{Les facteurs lourds}

Du temps de vacances disponible... En suedois, vancances prend le nom de "semester" ou "semestertid". Quoi de plus vrai que de se référer au terme semestre pour signifier une période relativement lon: gue d'arrêt du travail car les Suedois sont certes en avance à ce chapitre. La nouvelle loi sur les congés annuels donne droit a 5 semaines de vacances à tous les travailleurs. Les dispositions en matière de congés annuels ont évolué rapidement. Si on avait dejä, dès 1938, 12 jours de vacances payés, leur nombre passa en 1951 à 18, en 1963 à 4 semaines, et enfin en 1978 à 5 semaines. Ajoutons à cela les 12 jours de congés fériés, les congés de maladie, ceux reliés à

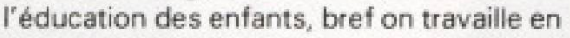
moyenne 1700 heures par an en Suède. II $y$ a donc longtemps que des conditions favorisant l'exercice des activités de loisir et de vacances sont en place. Les syndicats sont particulièrement revendicateurs en ce domaine. Alors qu'à $90 \%$ la main-d'oeuvre est syndiquée, il n'existe guère d'inégalités quant à la disposition d'un temps de vacances. Pour les années 80 , beaucoup s'accordent a dire que les Suédois pourraient disposer progressivement de 7 a 8 semaines de vacances.

La prise de vacances s'effectue en grande partie l'été, on le comprendra súrement. De part sa situation géographique, la Suède profite d'en moyenne 18 heures de clarté pendant la saison estivale. En Laponie, c'est la période du soleil de minuit. L'inverse est tout aussi vrai, l'hiver, alors que le temps est sombre et morose. L'été est en effet la perriode des grandes vacances par tradition. La loi oblige même que 4 semaines de vacances soient prises consécutivement. La semaine supplémentaire peut être prise à d'autres moments durant l'année. On peut également la conserver jusqu'à échéance de cinq ans, ce qui fait que le travailleur qui a accumulé à chaque année sa semaine supplémentaire, peut disposer au bout de cinq ans, de 9 semaines de vacances.
... un développement accéléré des résidences secondaires.

Le développement des résidences secondaires a rendu bon nombre de lieux inaccessi. bles à la pratique des loisirs et des vacances. Mème si on relève la construction de maisons secondaires sur les côtes est et ouest aussi tót qu'au $17 \mathrm{e}$ et $18 \mathrm{e}$ siècle, le phenomeme resta quelque peu exclusif jusqu'à la période d'entre-deux guerres. Cependant, à partir des années 30 , il y a eu un changement radical dans les habitudes de loisirs et de vacances des Suédois : on se rendait de plus en plus dans la nature, on construisait de plus en plus de maisons secondaires. Ce changement est lié à plu sieurs facteurs: une croissance continue du niveau de vie, une urbanisation rapide, les législations sur le temps de travail, le développement des voies de communications (les routes et les lignes ferroviaires), I'accès progressif à l'automobile. C'est dans les 20 dernières années que le phénomène s'est accentue de façon particulière:

\begin{tabular}{ll}
\hline $\begin{array}{l}\text { Tableau } 1 \\
\text { Nombre de maisons secondaires } \\
\text { (en chiftres arrondisi }\end{array}$ \\
Suede, 1957-1980 \\
1957 & 180,000 \\
1963 & 300,000 \\
1967 & 420,000 \\
1970 & 490,000 \\
1975 & 590,000 \\
1978 & 610,000 \\
1980 & 670,000
\end{tabular}

H. Fritidsbebrgglessens urveckling itrad statistiques sur les maisons secondairest 1957.19BO

La concentration dans les zones métropolitaines et les régions cótières est le portrait dominant de la distribution régionale des résidences secondaires. C'est, avant tout, I'attrait de l'eau (mer et grands lacs) et aussi la proximité de la résidence permanente qui influencent la localisation. Approximativement, $65 \%$ des résidences secondaires sont situées à moins de $1.5 \mathrm{~km}$ de la côte, d'un lac ou d'un cours d'eau majeur. 
La carte no 1 nous fait voir ces concentra tions le long de la cóte. Elles coincident, le plus souvent, avec les régions métropolitaines de Stockhoim, Goteborg et Malmo. L'ar. chipel de Stockholm où l'on trouve quelque 500 ïles a été propice à un tel développement. II n'est donc pas exagéré de dire que les zones métropolitaines et les villes de movennes dimensions sont toutes entourées d'une "ceinture" de résidences secondai res. En général, plus de la moitié de cellesci sont situees à une distance de $50 \mathrm{~km}$ ou moins de la résidence de leur propriétaire. Les $3 / 4$ sont à une distance de moins de $100 \mathrm{~km}$. Ce point est certainement caracté ristique de la Suède. Même si la résidence secondaire est modeste, on la veut le plus pres des lieux permanents d'habitat. $\mathrm{Ce}$ n'est pas tant le type, donc, que le nombre qui pose problème. Les données de 1980 indiquent qu"il y a 81 maisons secondaires par mille habitants, en ce pays! On estime que $20 \%$ de la population en possède une et que la moitié de la population urbaine peut en disposer d'une. Alors qu'un comité était chargé de définir les principales régions d'intereat national pour les loisirs de plein-air ainsi que les endroits, le long de la côte, qui se prêtent à la baignade, on s'est vite rendu compte que sur le total de la ligne côtière. $50 \%$ des endroits ne s'y prètent pas à cause des conditions naturelles et $40 \%$ sont inaccessibles à cause des constructions, surtout des résidences secondaires.

II apparait clairement qu'une augmentation même mineure de la construction de nouvelles résidences consommerait en très peu de temps la totalité de la côte.

Telle est le problematique qui entoure le débat à ce sujet en Suède. Alors qu'une population, de plus en plus concentrée en 3 zones métropolitaines et dans les villes movennes, dispose davantage de temps libre, l'accessibilité aux lieux de loisir est contrecarrée par le développement d'unités privees qui se transforment de plus en plus en résidences permanentes. Evoluant à un rythme rapide, ce point a certes intensifié les pressions pour qu'on précise les orientations futures car un tel changement de fonction suppose des coưts d'aménagement (électrification, egout, aqueduc) supérieurs a ce qu'il pourrait en coùter dans un milieu dẹjà urbanisé.

Comment, en effet, pouvait-on considérer que $20 \%$ de la population $115,2 \%$ avait effectivement utilisé une maison secondaire pour les vacances de 1980 ) occupe les meilleurs sites en contradiction évidente avec la règle du droit d'accès commun qui suppose que toute personne a droit à l'accès libre des lieux naturels.

La répartition des maisons secondaires en Suedde Inventaire 1975-76

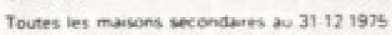

thelete

540

- $\quad 501900$

\section{Carte 1}

Aires d'aménagement pour la récréation

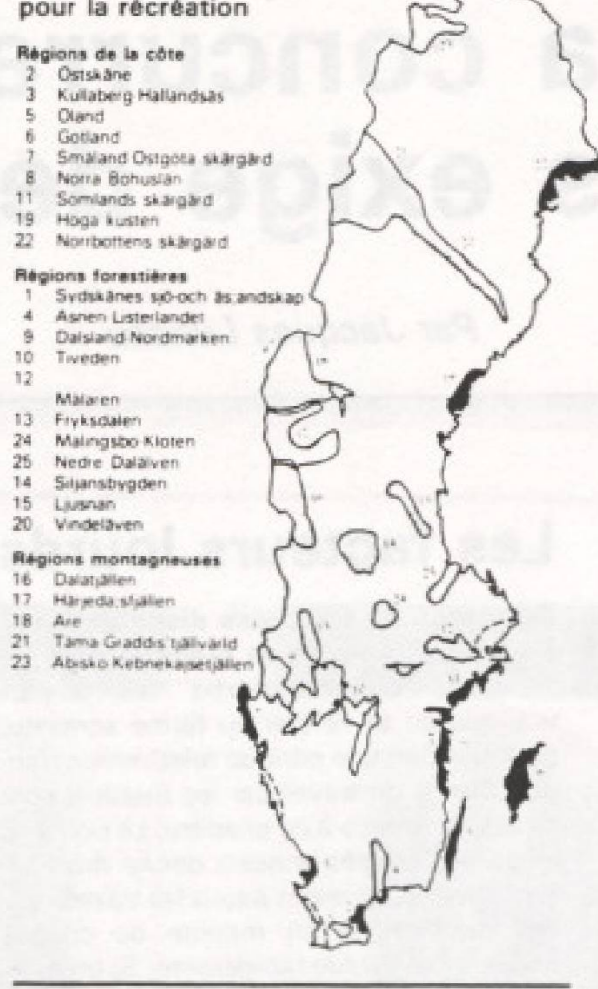

\section{Carte 2}

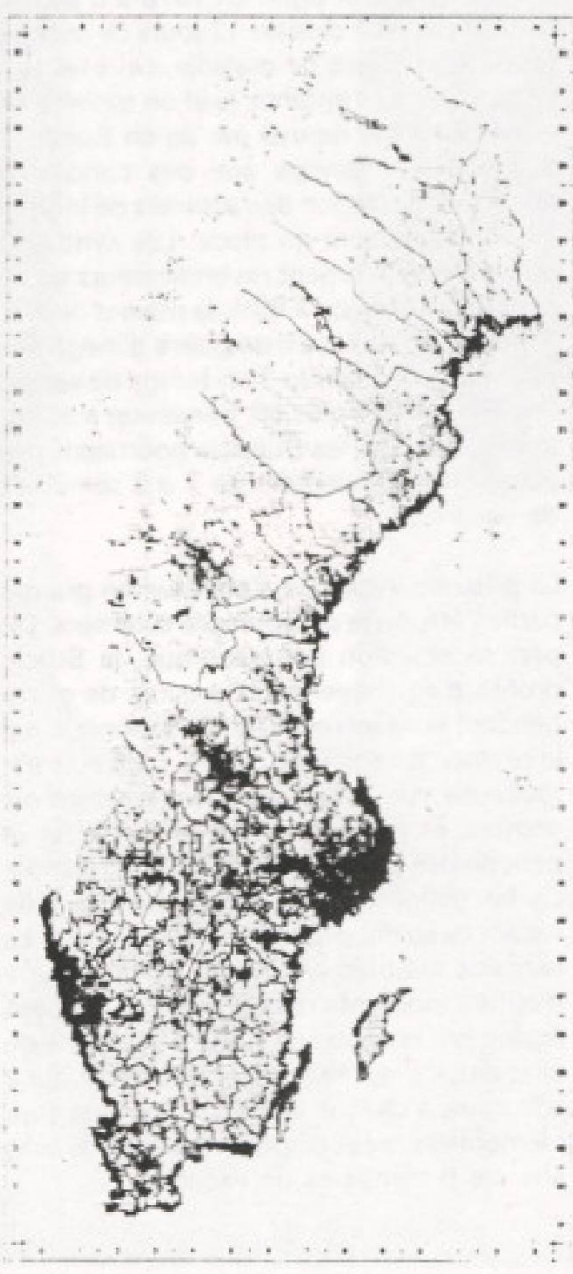

\section{Les actions entreprises}

Des aires d'aménagement de récréation

Si dejà l'A dministration nationale de la protection de l'environnement (Statens Naturvardserk) s'était, très tōt, prévalu de son pouvoir d'ériger des parcs nationaux, des resserves naturelles, son orientation en matière d'aménagement se limitait à la protection de la nature. Les travaux du comité chargé des questions d'environnement ${ }^{13}$ accentua une prise de conscience au sujet des aires de loisirs et de récréation à proximité des bassins de population. En 1975 une decision gouvernementale identifia 25 aires d'aménagement pour la récréation. La carte no 2 nous montre la localisation de ces aires d'aménagement. On peut voir que cer taines coincident avec les lieux ou la con centration de résidences secondaires ètait particulièrement élevée. Dans de telles zones, on interdit tout nouveau développe ment: celles le long de la côte et autour de certains lacs, sur les iles de Gotland et d'Oland et à proximité des principales régions urbaines.

Chaque annee, environ 9 millions de dollars sont investis et le programme d'aménage ment se poursuit de concert avec les col. lectivites locales. Une attention toute spe. ciale est accordée à l'accessibilité de ces lieux par les autorités des transports collec. tifs locaux.

Les types d'equipements

pour le futur

Amenager c'est prévoir. En raison des critiques largement formulees face au développement des maisons secondaires, un comité du ministêre du Logement, cette fois, s'est penché sur diffèrentes questions, à savoir: "Ouel type d'équipements sera en demande dans le futur?" "Quel type doit-on privilegier?"

Le rapport publie en 1982, privilégie, en matière d'amenagement touristique futur, deux types d'équipements: les sites de camping et les villages de vacances.

Les raisons évoquées sont simples: le peu d'espace que les collectivités locales disposent ne peut permettre l'accroissement du nombre de maisons secondaires. Des amé

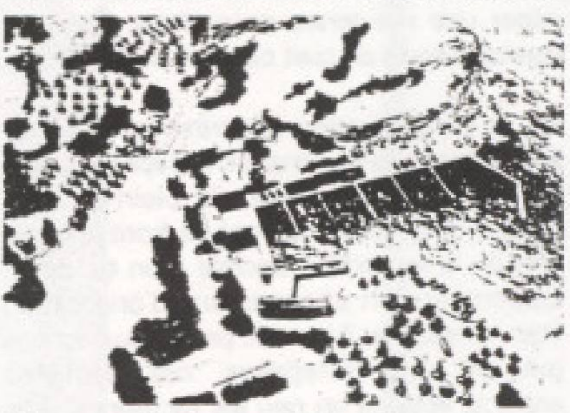

Un exemple d'un projet de village de vacaces, prẻs de Ertserod dans la commune de Tanums. 
nagements plus simples, pius concentrés et en locatif sont plus avantageux, à long terme, pour la commune qui veut investir dans le domaine du tourisme et des loisirs.

C'est, en effet, la commune qui est maitre d'oeuvre en la matière. Si déja $65 \%$ des terrains de camping sont sous proprièté des communes (les municipalités ici), $30 \%$ étant à l'entreprise privé, le programme d'immobilisation prévoit, en premier lieu, I'amélioration des équipements existants. On compte plus de 700 terrains de camping en Suède.

La construction de villages de vacances est sans aucun doute plus nouvelle. En 1955 , on n'en comptait que 21. Puis progressivement, le rythme de construction a augmente passant de 180 en 1972, 253 en 1981. En termes de capacité en lits, ceux-ci disposent de 25,000 lits environ.

Le croquis ci-contre présente un exemple de village de vacances qu'on voudrait bien voir augmenter en Suede. L'accent est mis sur l'amenagement intégré des ressources; les etablissements individuels y sont modestes et ceux, à vocation communautaire, plus imposants.

On prévoit dépenser près de 20 millions de dollars par an pour la réalisation de tels équipements. La participation du gouvernement central équivaut à $75 \%$. Les fonds sont destinés en premier lieu aux communes, aux conseils régionaux (landsting) et aux orga. nisations d'usagers intéressées à la realisa tion de tels equipements.

\section{Conclusion}

II n'y a pas lieu de croire que tout est réglé à ce sujet. Mème si des décisions importan tes ont été prises, des fonds substantiels attribués, il faudra voir comment l'interdiction de battir de nouvelles résidences secondaires, dans certaines zones, sera suivie. Comment, en général, la population vat-elle réagir? Vatelle délaisser ses habitudes individuelles de consommation et se tourner vers les equipements de type collectif? Comment les communes locales vont-elles accepter d'investir dans ce nouveau champ de compétences qui leur est en premier lieu attribué, notamment pour les villages de vacances? Comment concilier habitudes individuelles, pressions environnementales et souci de rentabilité?

Des points, tout aussi d'actualité ici au Quét bec comme la durée relativement courte de la saison touristique, I'ammortissement des equipements, suscitaient labas encore beaucoup d'interrogations. Mais, les Suédois ont pour trait de caractère de faire des réformes petit à petit sur une longue periode.

Loin d'être révolutionnaires, les changements sont évolutifs, le but étant de résoudre chaque problème au moment où il s'an- nonce. C'est dans cette perspective qu'on semblait entrevoir l'avenir au moment oủ jai quitté la Suède.

Le gouvernement entendait mettre l'accent sur ses nouvelles priorités certes, mais la concertation était de pair avec les interve. nants et la population en general afin que la collectivité suédoise toute entière fasse ses choix; choix qui vont marquer dans les prochaines années le portrait général du territoire suédois.

\section{Les intervenants en matière d'aménagement en Suède}

II ne faudrait pas passer sous silence que les paliers d'intervention en Suède ressem. blent beaucoup à ceux du Québec depuis la création récente des municipalités régio nales de comté.

Alors qu'on comptait environ 2,500 communes en 1862, une première réforme en 1952 réduisit le nombre a 1,037 . Plusieurs motifs la justifiaient; les migrations internes de la population, celle des campagnes s'intégrant aux agglomérations urbaines, mettaient les communautés rurales dans la quasi imposibilité de faire face aux nouvelles tåches qui les attendaient: l'éducation primaire et secondaire, les services sociaux, le logement etc

La réforme spécifiait que pas une commune ne devait compter moins de 2,000 habitants. En 1975, une nouvelle réforme était entre prise en fonction de l'indentification de pôles de croissance: une ville centrale et son hinterland. Le minimum de population etait alors de 8,000 habitants.

Aujourd hui on compte 279 communes dont plus de $40 \%$ ont une population exédant 20,000 habitants. Ce sont, en premier lieu, les communes locales qui sont responsables de l'aménagementiplans d'occupa: tion des sols, logement collectif, travaux publics). Les communes suédoises ont une politique d'acquisition des sols particulièrement active. Stockholm, la capitale, possede depuis 1920 pratiquement tous les terrains nécessaires à la construction domiciliaire.

Au niveau régional, il existe 24 "landsting" ou régions. C'est également un palier politique élu au suffrage universel. On s'y occupe principalement des services de santé (hopitaux) et du contröle de l'environnement.

Les conseils régionaux sont aussi le lieu de l'ajustement de l'administration centrale; le palier d'execution est majoritairement

\section{Notes}

1) Droit d'acces commun

Ce droit daccés commun n'est pas regle par la loi mais reteve du droin coutumier. II signifie que cha cun a le droit de se deplacer hbrement dims la nature et de fouler le sol drautrui, a condition de ne pas. endommager les biens du proprietaire

Tout le monde a le droit de cueillir les fruits sava ges, canoter sur les plans deau. Le camping libre est aussi permis meme sur les terrains prives en raison diune nuiter. decentralise a ce niveau. Un fonctionnaire nommé par le gouvernement central voit a la cohérence des actions du gouvernement II peut sieger au conseil régional elu mais n'a pas le droit de vote.

Le territoire est le mème tant pour l'admi nistration nationale que pour les conseils regionaux qui sont en fait le regroupement. en movenne, de 8 communes locales.

Au niveau central, differrents ministères et administrations nationales se chargent de l'amenagement. Mentionnons entre autres. le ministère de l'Agriculture via son admi. nistration nationale de la protection de l'environnement (Statens Naturvadsverk) et le ministère du Logement et de l'Aménage ment du territoire (Bostadsdepartementet et Statens Planverkl

Deux grandes legislations sont centrales en matière d'aménagement. La loi sur la pro tection de l'environnement promulgée en 1969 permettait à l'Etat central d'intervenit dans differents domaines: contröle de la pol lution, conservation de la nature, decisions concernant l'implantation des industries, etc

En 1972. le Parlement a adopté certaines directives générales, connues sous le nom de Plan national d'aménagement du ter. ritoire. Ce plan mettait en branle tout un processus de planification et d'aménage ment en continuelle interaction entre les communes locales et l'administration centrale.

Un vaste inventaire s'en est suivi, dans tous les domaines. Notons en particulier les macro-inventaires naturels, fauniques, culturels, historiques. C'est après cette phase que furent décrétées les aires d'amenage ment pour la récréation. Identifiant ces aires. d'intérèt national, c'est un comité du gou vernement lié au NVS qui se charge des réa lisations sur le terrain en collaboration avec les communes impliquées.

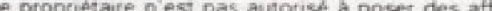
ches interd sant $\mathrm{I}$ acces notamment pour les plapes Un are de 300 metres en bordure de l eau est stric tement publique Chacun a droit de se baigne partout

2) "Frids Boende" Betankande Frantritidsboende Kom mitten Sou 1982 23. Stockhoim, 1982, 207 p Trad Maisens de loisirs. Rappon du comite denquete sut to question des masons de loisins

3) Primara Rekreationsomraden. Prop 1975 46 Komminten 